\title{
Effects of Quantum Spin-1/2 Impurities on the Magnetic Properties of Zigzag Spin Chains
}

\author{
Koushik Karmakar, ${ }^{1}$ Markos Skoulatos, ${ }^{2,3}$ Giacomo Prando, ${ }^{4,5}$ Bertran Roessli, ${ }^{2}$ Uwe Stuhr, ${ }^{2}$ \\ Franziska Hammerath, ${ }^{6,7}$ Christian Rüegg, ${ }^{2,8}$ and Surjeet Singh ${ }^{1, *}$ \\ ${ }^{1}$ Indian Institute of Science Education and Research, Pune, Maharashtra-411008, India \\ ${ }^{2}$ Laboratory for Neutron Scattering and Imaging, Paul Scherrer Institute, 5232 Villigen, Switzerland \\ ${ }^{3}$ Heinz, Maier-Leibnitz, Zentrum (MLZ) and Physics Department E21, Technische Universität München, D-85748 Garching, Germany \\ ${ }^{4}$ Center for Transport and Devices of Emergent Materials, TU Dresden, D-01062 Dresden, Germany \\ ${ }^{5}$ Leibniz-Institut für Festkörper- und Werkstoffforschung (IFW) Dresden, D-01171 Dresden, Germany \\ ${ }^{6}$ Dipartimento di Fisica and Unità CNISM di Pavia, I-27100 Pavia, Italy \\ ${ }^{7}$ Institute for Solid State Physics, Dresden Technical University, TU-Dresden, 01062 Dresden, Germany \\ ${ }^{8}$ Department of Quantum Matter Physics, University of Geneva, 1211 Geneva, Switzerland \\ (Received 13 April 2016; revised manuscript received 17 November 2016; published 7 March 2017)
}

We investigate the effect of $\mathrm{Co}^{2+}$ (spin-1/2) impurities on the magnetic ground state and low-lying spin excitations of the quasione-dimensional spin-1/2 antiferromagnet $\mathrm{SrCuO}_{2}$ by means of neutron scattering, muon spin spectroscopy, and bulk (ac and dc) magnetic susceptibilities. We found that dilute Co doping induces an Ising-like anisotropy and enhances the magnetic ordering temperature rather significantly, but preserves the gapless nature of the spin excitations. These results are in apparent contradiction with the recent studies of $\mathrm{Ni}$ (spin-1) doped $\mathrm{SrCuO}_{2}$. Low-temperature magnetic behavior of the Co-doped zigzag chains in $\mathrm{SrCuO}_{2}$ reveals the presence of a weak geometrical spin frustration.

DOI: 10.1103/PhysRevLett.118.107201

Low-dimensional quantum magnets show several unique and intriguing properties not found in their higher dimensional analogues [1]. Because of reduced dimensionality the quasiparticles in these systems are spatially confined, which makes them highly susceptible to any kind of disorder. Over the last few decades, this particular feature has been exploited successfully to uncover various exotic phenomena in low-dimensional spin systems $[2,3]$. In the Heisenberg antiferromagnetic (HAF) spin- $1 / 2$ chain where the quantum fluctuations are particularly pronounced, the ground state and low-lying spin excitations are qualitatively reorganized in the presence of disorder [4-10]. In the strong disorder limit, for example, the HAF spin- $1 / 2$ chain shows a universal RS phase in which the spins pair up to form nonoverlapping singlets over arbitrarily large separations, leading to a characteristic $T^{-\alpha}(\alpha<1)$ temperature dependence of the spin susceptibility $[5,6]$. In the dilute disorder limit, on the other hand, conformal field theories along with numerical renormalization group (RG) techniques predict interesting many-body quantum states arising due to strong interactions between the impurity spin and the collective spin degrees of freedom of the chain $[7,8]$. Some of these results have been confirmed in recent experiments. In particular, it has been shown that merely $1 \%$ of $\mathrm{Ni}$ (spin-1) impurity in the quasione-dimensional antiferromagnet $\mathrm{SrCuO}_{2}$ opens up a sizeable gap $(\sim 8 \mathrm{meV})$ in the spin excitation spectrum [10]. This gapping is proposed to be a manifestation of the Kondo-singlet state at the impurity site, predicted theoretically for an antiferromagnetically coupled spin-1 impurity in the spin-1/2 chain [7].
Interestingly, a spin-1/2 impurity shows a more complex renormalization behavior with the impurity spin either decoupled from the chain or overscreened via a twochannel Kondo effect $[8,9]$. However, experimental efforts to realize these and other interesting scenarios that may arise due to dilute doping are scarce. Here, we experimentally investigate the effect of $\mathrm{Co}^{2+}$ impurities with an effective spin $1 / 2$ in the prototypical HAF spin- $1 / 2$ chain compound $\mathrm{SrCuO}_{2}$. For comparison magnetic behavior of $\mathrm{Sr}_{2} \mathrm{CuO}_{3}$ is also investigated. Both these compounds show excellent one dimensionality because of their extremely small interchain $\left(J_{\perp}\right)$ to intrachain $(J)$ coupling ratio $\left(<10^{-3}\right)$ [11]. In particular, the large value of $J$ $(\sim 2000 \mathrm{~K})$ makes them ideal candidates to access the low-energy physics of HAF spin- $1 / 2$ chain. We show that the effect of $\mathrm{Co}^{2+}$ impurities (spin-1/2) is fundamentally different from the previously studied impurities $\mathrm{Zn}^{2+}$ (spin-0) and $\mathrm{Ni}^{2+}$ (spin-1) [10,12]. Co doping enhances the magnetic ordering and gives rise to a rich magnetic behavior that appears to be controlled by the Ising-like anisotropy of the $\mathrm{Co}^{2+}$ impurity. Unlike $\mathrm{Ni}$, the spin excitations also remain gapless upon Co doping.

Experiments were performed on the high-quality pristine and doped crystals with mosaic spread $<0.5^{\circ}$. Crystal growth and characterization details can be found in Refs. [13,14]. $\mathrm{SrCuO}_{2} \quad\left(\mathrm{Sr}_{2} \mathrm{CuO}_{3}\right)$ consists of zigzag (linear) spin- $1 / 2$ chains along the $c(b)$ axis of the orthorhombic unit cell. In the zigzag chain of $\mathrm{SrCuO}_{2}$, the nearest and next-nearest-neighbor $\mathrm{Cu}-\mathrm{O}-\mathrm{Cu}$ bond angles are $87.7^{\circ}$ and $180^{\circ}$, respectively [15]. The actual 


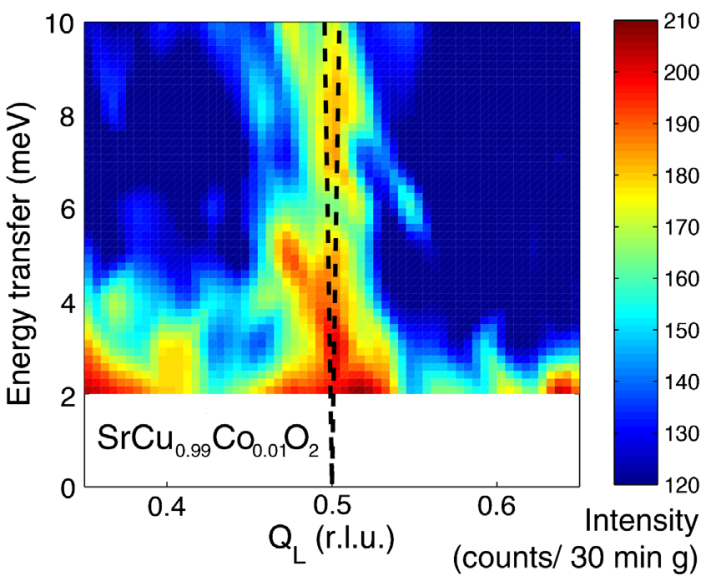

FIG. 1. Intensity color maps of the low-energy excitation spectrum for $\mathrm{SrCu}_{0.99} \mathrm{Co}_{0.01} \mathrm{O}_{2}$. The dashed lines correspond to the lower spinon boundary according to the "des CloizeauxPearson" analytical result [18] (see text for details).

Co concentration in the grown crystals was found to be roughly half the nominal value (hereafter, only nominal compositions are indicated). Neutron scattering experiments were performed at SINQ (Paul Scherrer Institute, Switzerland) on oriented single crystals weighing about $2 \mathrm{~g}$. Inelastic neutron scattering (INS) experiments were performed at $T=1.5 \mathrm{~K}$ using the thermal triple-axis spectrometer EIGER with final neutron energy $E_{f}=$ $14.7 \mathrm{meV}$ filtered using a graphite filter, while the elastic neutron scattering (ENS) experiments were carried out on the cold triple-axis spectrometer RITA-II, with final neutron energy $E_{f}=5 \mathrm{meV}$, using a Be filter to remove the $\lambda / 2$ contamination. Measurements of muon-spin spectroscopy $\left(\mu^{+} \mathrm{SR}\right)$ were performed at $\mathrm{S} \mu \mathrm{S}$ (PSI) on the GPS spectrometer $(\pi M 3$ beam line) in the temperature region $1.6 \mathrm{~K}<T<20 \mathrm{~K}$, in conditions of zero-magnetic field $\left(\mathrm{ZF}-\mu^{+} \mathrm{SR}\right)$ [16,17]. The dc magnetization and ac magnetic susceptibility were measured using a physical property measurement system (Quantum Design, USA).

Results of INS experiments on $1 \% \mathrm{Co}$-doped $\mathrm{SrCuO}_{2}$ are shown in Fig. 1. The vertical dashed lines originating at $Q_{L}=1 / 2$ indicate the des Cloiseaux-Pearson characteristic dispersion of spinon excitations [18], for intrachain coupling, $J=230 \mathrm{meV}$ [19]. Interestingly, the scattered intensity shows little variation as a function of the energy transfer, indicating a gapless spectrum down to the instrumental energy resolution of $2 \mathrm{meV}$. Contrary to this, $1 \% \mathrm{Ni}$-doped $\mathrm{SrCuO}_{2}$ was recently reported to exhibit a gapped behavior below $\sim 8 \mathrm{meV}$ (see Fig. 1 in Ref. [10]).

ENS data for $\mathrm{SrCu}_{0.99} \mathrm{Co}_{0.01} \mathrm{O}_{2}$ are presented in Fig. 2, where the magnetic scattering around $(1 / 2,0,1 / 2)$ at $T=1.5 \mathrm{~K}$ is displayed. Measurements were also performed at $T=20 \mathrm{~K}$, allowing us to subtract a weak, higher-order wavelength contamination. Panels (a) and (b) show the background-subtracted pure magnetic scattering along the $Q_{H}$ and $Q_{L}$ reciprocal lattice vectors,
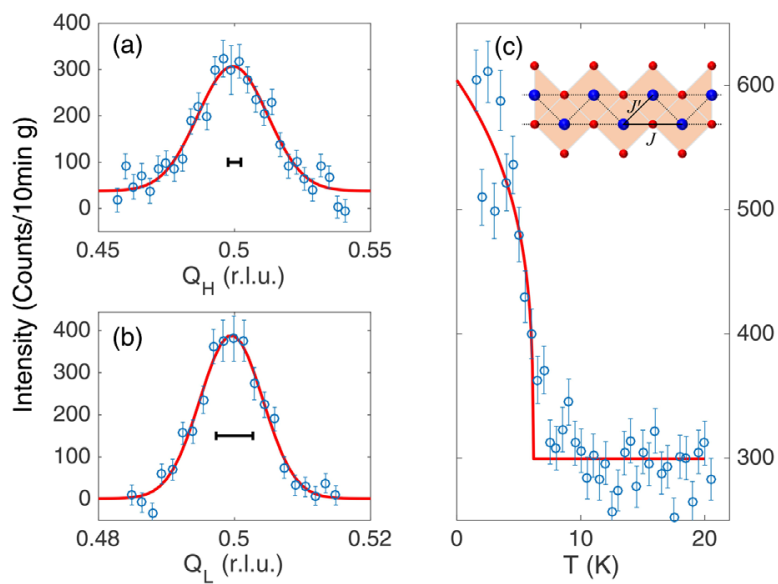

FIG. 2. Magnetic scattering of $\mathrm{SrCu}_{0.99} \mathrm{Co}_{0.01} \mathrm{O}_{2}$ at the $(1 / 201 / 2)$ Bragg peak. (a) and (b) depict the $Q_{H}$ and $Q_{L}$ dependence at $1.5 \mathrm{~K}$. Solid lines are Gaussian fits, while the horizontal bars refer to the instrumental resolution. (c) shows the order parameter as a function of $\mathrm{T}$, with a power-law fit (solid line) yielding $T_{N}=6.1(3) \mathrm{K}$. Inset: zigzag $\mathrm{Cu}$ (blue), $\mathrm{O}$ (red) chain with $180^{\circ}$ antiferromagnetic (AFM) superexchange $(J)$ and $90^{\circ}$ ferromagnetic $(\mathrm{FM})$ superexchange $\left(J^{\prime}\right)$.

respectively. These peaks are broadened in both directions contrary to a purely long-range order (LRO) magnetic phase, which is characterized by instrumental-resolutionlimited peak widths. The corresponding correlation lengths obtained are $\xi_{a}=44 \AA(\sim 12 a)$ and $\xi_{c}=155 \AA(\sim 40 c)$. Panel (c) shows the temperature dependence of the magnetic Bragg peak, with a power-law fit yielding $T_{N}=6.1(3) \mathrm{K}$. From the ENS results we infer that the $\mathrm{SrCu}_{0.99} \mathrm{Co}_{0.01} \mathrm{O}_{2}$ crystal exhibits an anisotropic quasi-LRO magnetic phase below $T \approx 6 \mathrm{~K}$. It should be remarked that due to Co doping the $T_{N}$ is sizeably enhanced from a value of $<2 \mathrm{~K}$ in the pristine compound [20], as opposed to the case of $\mathrm{Ni}$ doping where as little as $0.5 \%$ of $\mathrm{Ni}$ concentration destroys the magnetic ordering completely [21].

To further confirm the magnetic ordering we use $\mathrm{ZF}-\mu^{+} \mathrm{SR}[16,17]$, where the spin (de)polarization function $P_{z}(t)$ of implanted $\mu^{+}$is measured as a function of time $(t)$. In our measurements [22], the samples were aligned in order to have the $z$ direction parallel to the crystallographic $b$ axis, but with an arbitrary orientation of the $a-c$ plane. Experimental $P_{z}(t)$ curves for $\mathrm{SrCu}_{0.99} \mathrm{Co}_{0.01} \mathrm{O}_{2}$ are reported at representative $T$ values in Fig. 3. Curves were fitted using the function

$$
P_{z}(t)=\sum_{i} a_{T_{i}} \cos \left(\gamma B_{i} t\right) e^{-\sigma_{T_{i}}^{2} t^{2} / 2}+a_{L} e^{-\lambda_{L} t}
$$

usual for materials where a LRO magnetic phase is expected to develop [23]. Here, $\lambda_{L}$ is the longitudinal spin-lattice relaxation rate. Its $T$ dependence is shown in the inset of Fig. 3, displaying a narrow critical peak around the magnetic transition. At the same time, the development of 


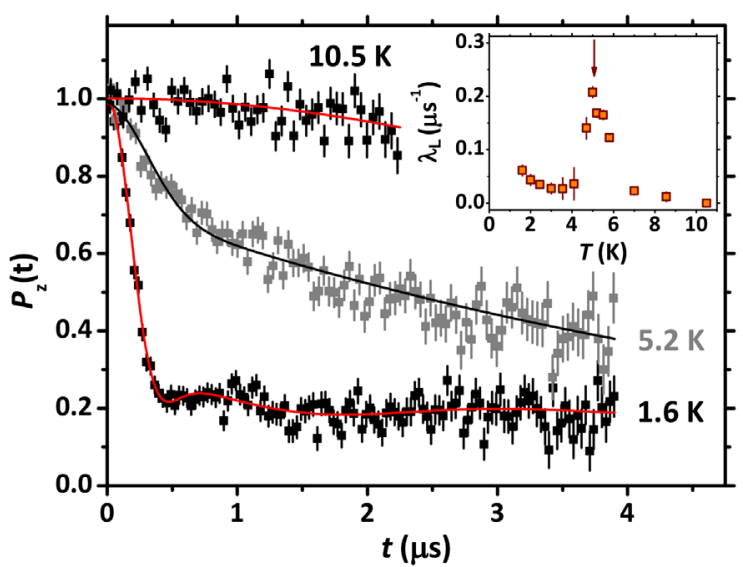

FIG. 3. (De)polarization $\left(P_{z}\right)$ as a function of time $(t)$ for representative temperatures $(T=1.6,5.2$, and $10.5 \mathrm{~K})$, shown in $\mathrm{ZF}$ conditions for $\mathrm{SrCu}_{0.99} \mathrm{Co}_{0.01} \mathrm{O}_{2}$. Continuous lines are best fits to the experimental data according to Eq. (1). Inset: $T$ dependence of the longitudinal relaxation rate. The arrow indicates the estimated $T_{N}$ value.

two $(i=1,2)$ highly damped, though visible, oscillations in the transversal fraction at low $T$ denotes a local homogeneous magnetic environment for the $\mu^{+}$whose distribution width $\sim \sigma_{T}$ is still comparable to the average $B$ value. These features further corroborate the quasi-LRO phase. Finally, in agreement with ENS data, orienting $z$ along the $a-c$ plane results in an anisotropic behavior of $P_{z}(t)$; these data will be discussed elsewhere in detail [22].

Figure 4(a) shows the temperature dependence of the dc magnetic susceptibility $(\chi)$ for $\mathrm{SrCu}_{0.99} \mathrm{Co}_{0.01} \mathrm{O}_{2} \cdot \chi$ along the crystallographic $a$ and $b$ axes $\left(\chi_{a}\right.$ and $\chi_{b}$, respectively) is comparable in magnitude with that of the pristine compound [13]. On the other hand, $\chi_{c}$ (parallel to the chains) is significantly enhanced and shows a well-defined peak at $T=5.4 \mathrm{~K}$. In the ordered state, a splitting of zerofield-cooled and field-cooled branches was also observed. We notice that $T_{N}$ is strongly dependent on the Co concentration, as shown in the inset of Fig. 4(a) where data from both dc magnetization and $\mu \mathrm{SR}$ [22] are shown. For comparison, $\chi$ of the linear chain compound $\mathrm{Sr}_{2} \mathrm{Cu}_{0.99} \mathrm{Co}_{0.01} \mathrm{O}_{3}$ with $H$ parallel to the chain is shown in Fig. 4(b). The data exhibit a sharp peak near $T=11 \mathrm{~K}$, which marks the onset of a magnetic LRO phase (confirmed using specific heat measurements not shown here). It is interesting to note that no thermomagnetic irreversibility in the susceptibility of linear chains could be detected down to the lowest temperature of $2 \mathrm{~K}$ in our experiments [Fig. 4(b)], which is a crucial difference compared to the zigzag chains under identical doping conditions. The upturn below $T=4.5 \mathrm{~K}$ is likely related to a second phase transition at even lower temperatures.

Isothermal magnetization of the two compounds at $T=2 \mathrm{~K}$ delineates these differences further. $M(H)$ of the zigzag chains exhibits a minor hysteresis loop [as shown
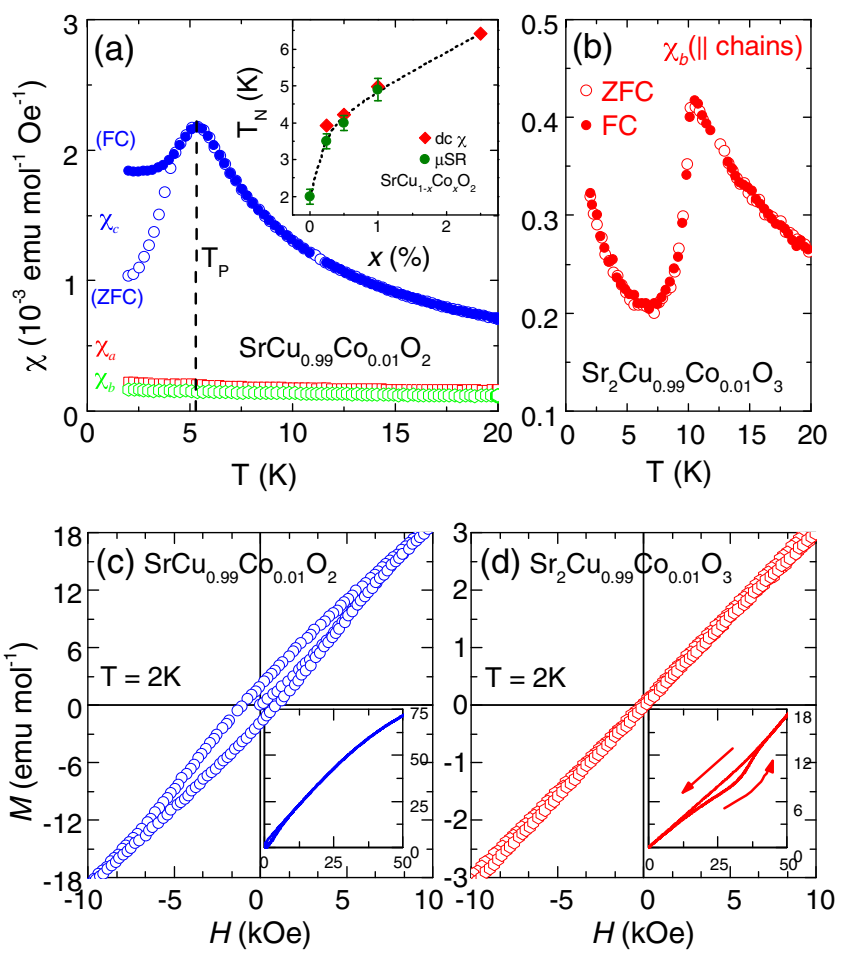

FIG. 4. Temperature dependent magnetic susceptibility of (a) $\mathrm{SrCu}_{0.99} \mathrm{Co}_{0.01} \mathrm{O}_{2}$ and (b) $\mathrm{Sr}_{2} \mathrm{Cu}_{0.99} \mathrm{Co}_{0.01} \mathrm{O}_{3}$. The inset in panel (a) shows the variation of $T_{N}$ with $\mathrm{Co}$ concentration. Isothermal magnetization of (c) $\mathrm{SrCu}_{0.99} \mathrm{Co}_{0.01} \mathrm{O}_{2}$ and (d) $\mathrm{Sr}_{2} \mathrm{Cu}_{0.99} \mathrm{Co}_{0.01} \mathrm{O}_{3}$ for fields applied parallel to the chains. Data over full ranges are shown in the insets. Arrows indicate the direction of field sweep.

in Fig. 4(c)] in line with the thermomagnetic irreversibility observed in the $M(T)$ behaviors. In the linear chain compound, on the other hand, no such hysteresis could be detected [see Fig. 4(d)]. Interestingly, $M(H)$ of $\mathrm{Sr}_{2} \mathrm{CuO}_{3}$ exhibits a metamagnetic transition near $30 \mathrm{kOe}$ with a small hysteresis [see the inset of Fig. 4(d)], not present in the undoped compound (not shown).

To characterize the quasilong-range-order phase of the zigzag chain, we show in Fig. 5(a) the temperature dependence of the real part $\left(\chi_{\mathrm{ac}}^{\prime}\right)$ of the ac magnetic susceptibility, measured by applying an alternating field $H_{\mathrm{ac}}=5$ Oe with frequency $(f)$ ranging from 100 to $10000 \mathrm{~Hz} . \chi_{\mathrm{ac}}^{\prime}$ signals at frequencies smaller than $100 \mathrm{~Hz}$ were extremely weak, and the imaginary part $\chi_{\mathrm{ac}}^{\prime \prime}$ was even weaker with poor signal-to-noise ratio over the entire frequency range. $\chi_{\mathrm{ac}}^{\prime}$ also exhibits a peak whose position, determined from the $d \chi^{\prime} / d T$ plots, shifts towards higher temperatures with increasing $f$, as is commonly observed in glassy systems [24]. The corresponding ratio $\Delta T_{f} / T_{f}$ was found to be around 0.04 per $\omega$ decade $(\omega=2 \pi f)$. This value is higher than what is typically reported for atomic spin glasses but smaller than the corresponding values reported for superparamagnets [25]. A more quantitative analysis can be performed by 

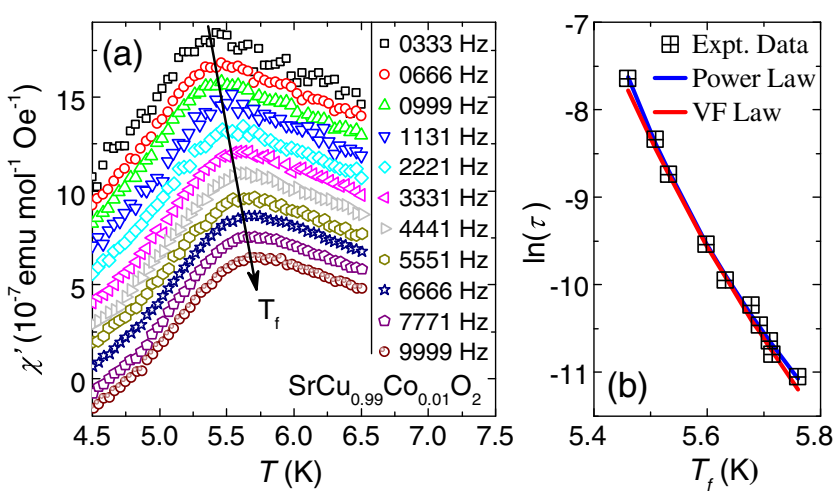

FIG. 5. (a) Temperature dependence of the real part of ac magnetic susceptibility $\left(\chi^{\prime}\right)$ of $\mathrm{SrCu}_{0.99} \mathrm{Co}_{0.01} \mathrm{O}_{2}$. (b) Relaxation time $(\tau)$ with respect to the peak temperatures. The solid lines are fit to the data (see the text for details). The curves in panel (a) are down shifted for clarity.

plotting $\ln (\tau)$ against the freezing temperature $\left(T_{f}\right)$ for various ac excitation frequencies $(f)$ [see Fig. 5(b)], where $\tau$ is the spin relaxation time that has been extracted from $f$ using the expression $\tau=(2 \pi f)^{-1}$. The data points were fitted using the theory of dynamical scaling near a phase transition [26], $\tau=\tau_{0}\left[\left(T_{f} / T_{g}\right)-1\right]^{-z \nu}$, where $\tau_{0}$ represents a microscopic characteristic time, $T_{g}$ is the spin glass temperature in the limit $f \rightarrow 0$, and $z \nu$ denotes the critical dynamical exponent. From the fitting, we get a value of $\tau_{0} \sim 10^{-10 \pm 1} \mathrm{~s}, z \nu \sim 4 \pm 1$, and $T_{g}=5.2 \pm 0.1 \mathrm{~K}$. The value of $T_{g}$ is in good agreement with the position of the peak in the dc magnetic susceptibility. For atomic spin glasses, the values of $\tau_{0}$ and of $z \nu$ typically lie in the range $10^{-13 \pm 1} \mathrm{~s}$ and $8 \pm 1$, respectively [26]. The values obtained here fall in the range that is more frequently reported for systems with inhomogeneous or short-range ordering $[27,28]$. An analogous analysis performed using the Vogel-Fulcher law $\tau=\tau_{0} \exp \left[\left(E_{a} / k_{B}\right) /\left(T-T_{0}\right)\right]$, which is typically used for glassy systems comprising short-range order, yielded a reasonably good fit by fixing the Vogel temperature $T_{0}\left(<T_{g}\right)$ and varying $\tau_{0}$ and the energy barrier $\left(E_{a}\right)$ as fitting parameters. For $T_{0}=4.2 \mathrm{~K}$, the values obtained for $\tau_{0}$ and $E_{a}$ are respectively $10^{-10 \pm 1} \mathrm{~s}$ and $20 \pm 2 \mathrm{~K}$. The value of the former parameter is in good agreement with the dynamical scaling approach.

As shown in the inset of Fig. 2(c), the zigzag topology of the chain gives rise to an additional ferromagnetic interaction $J^{\prime}(\approx 0.1 \times|J|[20])$ via the approximately $90^{\circ}$ $\mathrm{Cu}-\mathrm{O}-\mathrm{Cu}$ bond that couples the spins diagonally. The presence of $J^{\prime}$ frustrates the antiferromagnetic interaction $J$ within the chain [15]. As proposed by Haldane, if spin disorder is also incorporated in the frustrated zigzag chains, a glassy phase is favored [29], as is found to be the case here for Co-doped $\mathrm{SrCuO}_{2}$.

To summarize, we found that Co doping favors a magnetically ordered ground state with strongly enhanced ordering temperature (e.g., from $<2 \mathrm{~K}$ in $\mathrm{SrCuO}_{2}$ to $6 \mathrm{~K}$ in
$\mathrm{SrCu}_{0.99} \mathrm{Co}_{0.01} \mathrm{O}_{2}$ ) while preserving the gapless nature of the magnetic excitations. In contrast, doping with $\mathrm{Ni}$ not only opens a sizeable spin gap but also suppresses the magnetic ordering $[10,12,21]$. In the $\mathrm{Ni}$ case, the spinsinglet formation at the impurity sites disrupts the translation invariance of the chain locally, yielding a spin pseudogap whose size scales with the impurity concentration [7]. Recent NMR results support this hypothesis [30]. Suppression of magnetic order is also a result of the finite-size effects due to severing of the correlations along the chain [31]. The rate of suppression of $\mathrm{T}_{N}$ for low concentrations of $\mathrm{Ni}$ doping was also shown to be in good agreement with the theory [21]. Evidently, the behavior of Co-doped chains cannot be captured using these theories.

To understand the behavior of $\mathrm{Co}^{2+}$ impurity, we recall that in a square-planar coordination $\mathrm{Co}^{2+}\left(3 d^{8}\right)$ the ion has a Kramer's doublet ground state with an effective spin $1 / 2$ and an Ising-like anisotropy [32,33]. If one disregards this anisotropy, $\mathrm{Co}^{2+}$ doping would be analogous to the case of spin- $1 / 2$ impurity in the spin- $1 / 2$ chain theoretically analyzed by Eggert et al. [8]. Depending upon the sign of the coupling (ferromagnetic or antiferromagnetic) between the impurity spin and the host spins, the fixed points of the RG flow in this case correspond either to a broken chain with a decoupled impurity spin (ferromagnetic case) or to an overscreened impurity spin in a periodic chain in analogy with the two-channel Kondo effect (antiferromagnetic case). In the latter case, the low-energy behavior of the renormalized chain is expected to be non-Fermi-liquidlike with a logarithmically diverging spin susceptibility [8,9]. Since the measured excitations remained gapless upon $\mathrm{Co}^{2+}$ doping, the impurity spins do not seem to disrupt the periodicity of the chain as expected in the antiferromagnetic case above. However, the pronounced increase of the ordering temperature and the anisotropic magnetic response upon Co doping cannot be accounted for using this theory-pointing at the importance of the single-ion anisotropy of the doped $\mathrm{Co}^{2+}$ ion. Indeed, the Co-doped chains appear to have behavioral similarities with the prototypal spin-1/2 Ising-chain compounds $\mathrm{BaCo}_{2} \mathrm{~V}_{2} \mathrm{O}_{8}$ and $\mathrm{SrCo}_{2} \mathrm{~V}_{2} O_{8}$, which show a Néel-type ordering and field-induced transitions in conformity with the spin-1/2 XXZ model [34,35].

It is intriguing that as small as $0.25 \%$ of Co doping (the smallest concentration investigated) augments the ordering and switches the bulk behavior from Heisenberg to Isinglike. Since the spin correlations in the spin- $1 / 2$ chain decay only as a power law, the distant $\mathrm{Co}^{2+}$ spins in the doped chain probably interact weakly via the intervening $\mathrm{Cu}^{2+}$ spins, favoring the Néel-type ordering as in the $X X Z$ model. In this model, above a critical longitudinal field, a novel incommensurate spin-density wave phase replaces the Néel order via a first-order transition [35-37]. The hysteretic metamagnetic transition in the $M(H)$ plot of $\mathrm{Sr}_{2} \mathrm{Cu}_{0.99} \mathrm{Co}_{0.01} \mathrm{O}_{3}$ (Fig. 4) is consistent with this picture. 
An analogous transition was also reported for the aforementioned Ising-chain compounds [38,39]. The metamagnetic transition is not seen for Co-doped $\mathrm{SrCuO}_{2}$, probably due to the additional spin frustration term in the Hamiltonian.

To conclude, we showed that Co impurity has a rather strong and unexpected effect on the magnetic behavior of weakly coupled antiferromagnetic spin- $1 / 2$ chains. Dilute doping with Co leads to highly anisotropic magnetization and a significantly enhanced magnetic ordering temperature, contrary to $\mathrm{Ni}$ or $\mathrm{Zn}$ doping studied previously. These findings show that the ground state and magnetic excitations of the antiferromagnetic spin- $1 / 2$ chain depend sensitively on the spin state and symmetry properties of the doped impurity. This work should motivate further theoretical investigations on Ising spin-1/2 impurity in the spin- $1 / 2$ chains.

S. S. and C. R. acknowledge travel support through IndoSwiss personnel exchange program under Grant No. INT/ SWISS/ISJRP/PEP/P-06/2012. M. S. acknowledges financial support through FP7/2007-2013 under Grants No. 290605 and No. TRR80 of DFG. G. P. acknowledges support by the Research Fellowship for Postdoctoral researchers of the Alexander von Humboldt Stiftung. G. P. and F. H. acknowledge support by the Sonderforschungsbereich (SFB) 1143 project granted by the Deutsche Forschungsgemeinschaft (DFG).

*surjeet.singh@iiserpune.ac.in

[1] T. Giamarchi, Quantum Physics in One Dimension, International Series of Monographs on Physics (Clarendon Press, Oxford, 2003).

[2] H. Alloul, J. Bobroff, M. Gabay, and P. J. Hirschfeld, Rev. Mod. Phys. 81, 45 (2009).

[3] A. Zheludev and T. Roscilde, C.R. Phys. 14, 740 (2013).

[4] S. K. Ma, C. Dasgupta, and C. K. Hu, Phys. Rev. Lett. 43, 1434 (1979).

[5] D. S. Fisher, Phys. Rev. B 50, 3799 (1994).

[6] T. Shiroka, F. Casola, W. Lorenz, K. Prša, A. Zheludev, H.-R. Ott, and J. Mesot, Phys. Rev. B 88, 054422 (2013).

[7] S. Eggert and I. Affleck, Phys. Rev. B 46, 10866 (1992).

[8] S. Eggert, D. P. Gustafsson, and S. Rommer, Phys. Rev. Lett. 86, 516 (2001).

[9] D. G. Clarke, T. Giamarchi, and B. I. Shraiman, Phys. Rev. B 48, 7070 (1993).

[10] G. Simutis, S. Gvasaliya, M. Månsson, A. L. Chernyshev, A. Mohan, S. Singh, C. Hess, A. T. Savici, A. I. Kolesnikov, A. Piovano, T. Perring, I. Zaliznyak, B. Büchner, and A. Zheludev, Phys. Rev. Lett. 111, 067204 (2013).

[11] N. Motoyama, H. Eisaki, and S. Uchida, Phys. Rev. Lett. 76, 3212 (1996).

[12] K. Karmakar and S. Singh, Phys. Rev. B 91, 224401 (2015).

[13] K. Karmakar, A. Singh, S. Singh, A. Poole, and C. Rüegg, Cryst. Growth Des. 14, 1184 (2014).

[14] K. Karmakar, R. Bag, and S. Singh, Cryst. Growth Des. 15, 4843 (2015).
[15] I. A. Zaliznyak, C. Broholm, M. Kibune, M. Nohara, and H. Takagi, Phys. Rev. Lett. 83, 5370 (1999).

[16] S. J. Blundell, Contemp. Phys. 40, 175 (1999).

[17] A. Yaouanc and P. Dalmas de Réotier, Muon Spin Rotation, Relaxation, and Resonance: Applications to Condensed Matter (Oxford University Press, Oxford, 2011).

[18] J. des Cloizeaux and J. J. Pearson, Phys. Rev. 128, 2131 (1962).

[19] H. Rosner, H. Eschrig, R. Hayn, S. L. Drechsler, and J. Malek, Phys. Rev. B 56, 3402 (1997).

[20] M. Matsuda, K. Katsumata, K. M. Kojima, M. Larkin, G. M. Luke, J. Merrin, B. Nachumi, Y. J. Uemura, H. Eisaki, N. Motoyama, S. Uchida, and G. Shirane, Phys. Rev. B 55, R11953 (1997).

[21] G. Simutis, M. Thede, R. Saint-Martin, A. Mohan, C. Baines, Z. Guguchia, R. Khasanov, C. Hess, A. Revcolevschi, B. Büchner, and A. Zheludev, Phys. Rev. B 93, 214430 (2016).

[22] G. Prando (to be published).

[23] G. Prando, O. Vakaliuk, S. Sanna, G. Lamura, T. Shiroka, P. Bonfà, P. Carretta, R. De Renzi, H.-H. Klauss, C. G. F. Blum, S. Wurmehl, C. Hess, and B. Büchner, Phys. Rev. B 87, 174519 (2013).

[24] M. Charilaou, J. F. Löffler, and A. U. Gehring, Phys. Rev. B 83, 224414 (2011).

[25] J. A. Mydosh, Spin Glasses: An Experimental Introduction (Taylor \& Francis, London, 1993).

[26] R. Mathieu, D. Akahoshi, A. Asamitsu, Y. Tomioka, and Y. Tokura, Phys. Rev. Lett. 93, 227202 (2004).

[27] N. Hanasaki, K. Watanabe, T. Ohtsuka, I. Kézsmárki, S. Iguchi, S. Miyasaka, and Y. Tokura, Phys. Rev. Lett. 99, 086401 (2007).

[28] K. De, M. Patra, S. Majumdar, and S. Giri, J. Phys. D 40, 7614 (2007).

[29] F. D. M. Haldane, Phys. Rev. Lett. 61, 1029 (1988).

[30] Y. Utz, F. Hammerath, S. Nishimoto, C. Hess, N. S. Beesetty, R. Saint-Martin, A. Revcolevschi, B. Büchner, and H.-J. Grafe, Phys. Rev. B 92, 060405 (2015).

[31] S. Eggert, I. Affleck, and M. D. P. Horton, Phys. Rev. Lett. 89, 047202 (2002).

[32] A. Abragam and M. H. L. Pryce, Proc. R. Soc. A 206, 173 (1951)

[33] J. C. Slonczewski, J. Appl. Phys. 32, S253 (1961).

[34] A. K. Bera, B. Lake, W. D. Stein, and S. Zander, Phys. Rev. B 89, 094402 (2014).

[35] S. Kimura, M. Matsuda, T. Masuda, S. Hondo, K. Kaneko, N. Metoki, M. Hagiwara, T. Takeuchi, K. Okunishi, Z. He, K. Kindo, T. Taniyama, and M. Itoh, Phys. Rev. Lett. 101, 207201 (2008).

[36] S. Kimura, T. Takeuchi, K. Okunishi, M. Hagiwara, Z. He, K. Kindo, T. Taniyama, and M. Itoh, Phys. Rev. Lett. 100, 057202 (2008).

[37] T. Suzuki, N. Kawashima, and K. Okunishi, J. Phys. Soc. Jpn. 76, 123707 (2007).

[38] S. Kimura, H. Yashiro, M. Hagiwara, K. Okunishi, K. Kindo, Z. He, T. Taniyama, and M. Itoh, J. Phys. Conf. Ser. 51, 99 (2006).

[39] A. Okutani, T. Kida, T. Usui, T. Kimura, K. Okunishi, and M. Hagiwara, in 20th International Conference on Magnetism (ICM), 2015 (Elsevier, New York, 2015); Phys. Procedia 75, 779 (2015). 